\title{
GC-MS analysis of rhizome ethanol extracts from Curcuma aeruginosa accessions and their efficiency activities as anticancer agent
}

\author{
WARAS NURCHOLIS ${ }^{1,2, \boldsymbol{v}}$, NURUL KHUMAIDA ${ }^{2}$, MARIA BINTANG ${ }^{3}$, MUHAMAD SYUKUR ${ }^{3}$ \\ ${ }^{1}$ Tropical Biopharmaca Research Center, Institut Pertanian Bogor. Jl. Taman Kencana No. 3, Kampus IPB Taman Kencana, Bogor 16128, West Java, \\ Indonesia. Tel./fax.: +62-251-8423267, `email: wnurcholis@apps.ipb.ac.id \\ ${ }^{2}$ Department of Biochemistry, Faculty of Mathematics and Natural Sciences, Institut Pertanian Bogor. J1. Agathis, Kampus IPB Dramaga, Bogor 16680, \\ West Java, Indonesia \\ ${ }^{3}$ Department of Agronomy and Horticulture, Faculty of Agriculture, Institut Pertanian Bogor. Jl. Meranti, Kampus IPB Dramaga, Bogor 16680, West \\ Java, Indonesia
}

Manuscript received: 3 February 2021. Revision accepted: 8 February 2021.

\begin{abstract}
Nurcholis W, Khumaida N, Bintang M, Syukur M. 2021. GC-MS analysis of rhizome ethanol extracts from Curcuma aeruginosa accessions and their efficiency activities as anticancer agent. Biodiversitas 22: 1179-1186. This work aimed to evaluate the bioactive compounds and anticancer activity in rhizome extract of ten Curcuma aeruginosa accessions to explore their pharmacological values. The GC-MS analysis was used to identify bioactive compounds. The cytotoxic activity was performed against MCF-7 (Human breastadeno carcinoma) and Vero cell lines using MTT assay. The GC-MS analysis revealed 71 of the compounds as sesquiterpenes (36), monoterpenes (20), phenolics (5), diterpenes (4), phenanthrene (1), tetrapeptides (1), oxazole (1), triazine (1), piperidine (1), and oxygenated hydrocarbons (1). The isocurcumenol was the most dominant metabolite in ethanol extract of $C$. aeruginosa rhizome, with the highest produced by KP accession (22.01\%) followed MD accession (21.12\%). However, camphor and $\beta$-elemene were the metabolites produced by all accessions studied. In the Vero cell line as a normal cell, the cytotoxic activity varied from $13.28 \%$ (MD) to $45.17 \%$ (PW). Furthermore, the cytotoxic activity ranged from $1.16 \%$ (LC) to $49.70 \%$ (MD) against the MCF-7 cell line. The highest anticancer activity was produced in MD accessions; thus, it can be used as a source of quality raw materials for the pharmace utical and food industry. Besides that, it can also be further developed to obtain superior varieties through plant breeding programs.
\end{abstract}

Keywords: Agricultural biochemistry, bioactivities, chemometric analysis, profiling metabolite, volatile compounds

\section{INTRODUCTION}

Curcuma aeruginosa, a perennial plant belonging to the Zingiberaceae family, is used as medicinal plant. It is a native of Myanmar and distributed to Indonesia, Malaysia, Indochina, and Ceylon (Sirirugsa et al. 2007). In traditional medicine, $C$. aeruginosa is used to treat many diseases such as flatulence, dysentery, dyspepsia, and gastritis (Theanphong et al. 2015). The C. aeruginosa plant contains many biologically active compounds obtained from rhizomes and leaves, used as a possible medicine for various human diseases (Moektiwardoyo et al. 2014; Srivilai et al. 2018; Suphrom et al. 2012). The phytochemical compounds contained in the $C$. aeruginosa contribute to its pharmacological properties. C. aeruginosa contains several bioactive compounds such as flavonoid, phenolic acids (Nurcholis et al. 2016b), curcumin, dimethoxycurcumin and bisdemethoxycurcumin (Nurcholis et al. 2019, 2016a), germacrone (Hossain et al. 2015; Srivilai et al. 2018), isocurcumenol, zederone, curcumenol, dehydrocurdione, zedoarondiol (Suphrom et al. 2012), tropolone, eucalyptol, and curcumol (Fitria et al. 2019). The reported biological activities of $C$. aeruginosa and its metabolites include antimicrobial (Akarchariya et al. 2017), analgesic effect (Reanmongkol et al. 2006), anti-androgenic (Suphrom et al. 2012), uterine relaxant effect (Thaina et al. 2009), antioxidant (Nurcholis et al. 2017), antinociceptive
(Hossain et al. 2015), hair growth promotor (Pumthong et al. 2012), and anticancer (Fitria et al. 2019). Therefore, it is crucial to select $C$. aeruginosa accessions with high phytochemical content and high pharmacological activities, which can be developed for plant breeding programs.

The composition and bioactive contents of medicinal plants can be affected by different factors, including genotypes (Batubara et al. 2020) and environmental factors (Mahajan et al. 2020; Ncube et al. 2012). The polyphenol and curcuminoids contents of $C$. aeruginosa accessions are varied by geographic location (Nurcholis et al. 2016b, 2016a), but whether this variation is due to environmental differences or genetic variability is unclear. Recently, the GC-MS has been well established to identify different metabolites from plant extracts (Ghimire et al. 2017; Ukwubile et al. 2019). GC-MS analysis of C. aeruginosa essential oils identified monoterpenes and sesquiterpenes compounds associated with antibacterial activity (Akarchariya et al. 2017). Several metabolites such as terpenoids, organic acids, sterols, sugars, and fatty acids have been reported from the different extracts (methyl tertbutyl ether, methanol/chloroform) of $C$. aeruginosa using GC-MS analysis (Simoh and Zainal 2015). However, the identification of metabolites from $C$. aeruginosa accessions in ethanol extract associated with an anticancer activity using GC-MS and chemometric analyses is considerably limited. 
Due to the importance of $C$. aeruginosa extract, it is necessary to investigate metabolites composition across various accessions related to pharmacological activities. Therefore, the present research focused on metabolites identification from the ethanol extract of different $C$. aeruginosa accessions and further evaluated for anticancer activity against MCF-7 cell line. We used the same soil and environment for the growth of $C$. aeruginosa rhizome; thus, the different accession results are a direct reflection of the genetic diversity. Profiling metabolites and cytotoxicity data were used to classify $C$. aeruginosa accessions using chemometric analysis. This result also showed how to choose the elite accessions to develop commercially grown varieties of $C$. aeruginosa.

\section{MATERIALS AND METHODS}

\section{Plant material and extraction}

A total of $10 \mathrm{C}$. aeruginosa fresh rhizome accessions obtained from various regions of Indonesia were collected in February 2015 and further identified by expert from the Tropical Biopharmaca Research Center, IPB University, Indonesia (Table 1). The rhizome of sample collection was then cultivated at the Tropical Biopharmaca Research Center in Bogor City (10642'53.22" E, 6³2'25.47" N), West Java Province, in Indonesia at an altitude of $142.60 \mathrm{~m}$ and arranged with three replications using a completely randomized design. Rhizome samples were planted in the spacing of $50 \mathrm{~cm} \times 50 \mathrm{~cm}$ and grown under the same conditions in latosol soil with contained $0.15 \% \mathrm{~N}, 1.52 \%$ organic $\mathrm{C}$, and $\mathrm{pH}$ of $4.5-5$. The soil was prepared by $1 \mathrm{~kg}$ of cow manure per planting hole two weeks before planting. All rhizomes were harvested at nine months after planting in August 2016. In this work, all fresh rhizomes were cut and dried until the moisture content was $\leq 10 \%$ and when subjected to extraction, powdered at 80 mesh.

The rhizome powder was extracted using the maceration technique described in our previous research (Nurcholis et al. 2015). Briefly, the sample powder (25 g) were extracted at room temperature with $70 \%$ ethanol of $250 \mathrm{ml}$ for $24 \mathrm{~h}$. The solution sample was filtered with the Whatman filter paper of number 4 . Then, the solution was evaporated at $50^{\circ} \mathrm{C}$ using a rotary evaporator (BUCHI, R250, Switzerland). The extracted result was used for GCMS and anticancer analysis.

\section{GC-MS analysis}

The ethanol extract of each accession was analyzed for the metabolite profile using gas chromatography-mass spectrometry (GC-MS). Previously, the sample extract (1 g) was extracted with $10 \mathrm{ml}$ of hexane and then sonicated for $30 \mathrm{~min}$. The filtered hexane solution $(2 \mu \mathrm{l})$ was used for GC-MS analysis (Agilent GC 7890 series and Agilent MS 6950 series, USA) equipped with HP-5ms GC J\&W capillary column $(30 \mathrm{~m} \times 0.25 \mathrm{~mm}$ i.d. and $0.25 \mu \mathrm{m}$ film thickness). The helium was used as carrier gas with a 1 $\mathrm{ml} / \mathrm{min}$ flow rate and injector temperature $250^{\circ} \mathrm{C}$. The column oven temperature was programmed as follows: $40^{\circ} \mathrm{C}$ (hold for $2 \mathrm{~min}$ ) to $50^{\circ} \mathrm{C} / \mathrm{min}$ to $280^{\circ} \mathrm{C}$ as final temperature (hold for $2 \mathrm{~min}$ ). The MS was operated at 70 $\mathrm{eV}$, and the mass range scanned was 35 - $500 \mathrm{amu}$. All accessions were analyzed once without replication. The identification of compounds, including name, chemical structure, and molecular weight, was determined by adjusting the chromatogram spectra peaks with the known compounds in the NIST databases and PubChem data.

\section{MTT assay}

The anticancer analysis was performed using breast cancer cell line MCF-7 (ATCC HTB 22) and normal Vero cells (ATCC CCL 81) as a comparison. The cytotoxic activity of sample extract was measured colorimetrically using MTT (3- (4,5-dimethylthiazol-2-yl) -2,5-diphenyltetrazolium bromide) (Sigma-Aldrich, St. Louis, MO, USA) according to (Vijayarathna and Sasidharan 2012) protocols with slight modification. The MTT assay is often used to measure how cells are alive and to detect the toxicity of cells. This test is attributed to the loss of a yellow tetrazolium salt (MTT) to purple formazan crystals by metabolically active cells. Briefly, the MCF-7 and Vero cells were cultured in Dulbecco's minimum Eagle's medium (Gibco, Rockville, MD, USA) and supplemented with fetal bovine serum (5\%; Sigma-Aldrich, St. Louis, MO, USA), $100 \mu \mathrm{g} / \mathrm{mL}$ penicillin (Gibco, Rockville, MD, USA) and $100 \mu \mathrm{g} / \mathrm{mL}$ streptomycin (Gibco, Rockville, MD, USA). Cells were grown at a concentration of 5000 cells in $100 \mu \mathrm{L}$ of growth medium. The extract $(250 \mu \mathrm{g} / \mathrm{ml})$ was added after the cells reached $50 \%$ confluent ( 24 hours). The MTT test was carried out on the third day by adding $10 \mu \mathrm{L}$ of MTT per test well and incubating 4 hours at $37^{\circ} \mathrm{C}$. Formazan crystals dissolved in ethanol. The absorbance value reading was carried out at a wavelength of $595 \mathrm{~nm}$ with a microplate reader (Bio-Rad 680, USA). Cytotoxicity value was calculated based on the percentage of inhibition of cell growth.

Table 1. The location origin and name of the ten C. aeruginosa accessions.

\begin{tabular}{lcccc}
\hline \multicolumn{1}{c}{ Origin/Location } & \multirow{2}{*}{ Accession code } & \multicolumn{3}{c}{ Geographical information } \\
\cline { 3 - 5 } & & Altitude (m) & Latitude (N) & Longitude (E) \\
\hline Klewer, Central Java & KL & 96 & $7^{\circ} 35^{\prime} 05.66^{\prime \prime}$ & $110^{\circ} 49^{\prime} 45.38^{\prime \prime}$ \\
Pakem, Yogyakarta & PK & 424 & $7^{\circ} 39^{\prime} 55.46^{\prime \prime}$ & $110^{\circ} 25^{\prime} 11.30^{\prime \prime}$ \\
Beringharjo, Yogyakarta & BH & 115 & $7^{\circ} 47^{\prime} 56.40^{\prime \prime}$ & $110^{\circ} 22^{\prime} 01.56^{\prime \prime}$ \\
Gunung Kidul, Yogyakarta & GK & 180 & $7^{\circ} 58^{\prime} 04.87^{\prime \prime}$ & $110^{\circ} 36^{\prime} 09.67^{\prime \prime}$ \\
Kulonprogo, Yogyakarta & KP & 20 & $7^{\circ} 56^{\prime} 25.03^{\prime \prime}$ & $110^{\circ} 14^{\prime} 20.30^{\prime \prime}$ \\
Purworejo, Central Java & PW & 56 & $7^{\circ} 44^{\prime} 25.35^{\prime \prime}$ & $110^{\circ} 01^{\prime \prime} 59.00^{\prime \prime}$ \\
Madura, East Java & MD & 4 & $7^{\circ} 02^{\prime} 48.90^{\prime \prime}$ & $112^{\circ} 43^{\prime} 47.32^{\prime \prime}$ \\
Cirebon, West Java & LC & 1 & $6^{\circ} 48^{\prime} 17.09^{\prime \prime}$ & $108^{\circ} 48^{\prime} 06.04^{\prime \prime}$ \\
Bogor, West Java & CB & 148 & $6^{\circ} 32^{\prime} 35.89^{\prime \prime}$ & $106^{\circ} 41^{\prime} 22.41^{\prime \prime}$ \\
Muara Bungo, Jambi & MB & 65 & $1^{\circ} 37^{\prime} 00.61^{\prime \prime}$ & $102^{\circ} 22^{\prime} 16.28^{\prime \prime}$ \\
\hline
\end{tabular}




\section{Data analysis}

Statistical analysis of antiproliferative data was performed by analysis of variance (ANOVA) followed by the Scott-Knott test to identify significant differences between $C$. aeruginosa accessions with $\mathrm{R}$ using package 'ExpDes'. Significant differences between accessions were determined at $\mathrm{p} \leq 0.05$. $\mathrm{R}$ was used with package 'pheatmap' and 'factoextra' for hierarchical cluster analysis (HCA)-heatmap dendrogram and principal component analysis (PCA), respectively. Graph of figure was generated using GraphPad Prism 8 for masOS (GraphPad Software Inc., San Diego, California, USA) Version 8.4.3.

\section{RESULTS AND DISCUSSION}

\section{Metabolite compositions}

Seventy-one metabolite compounds in the ethanolic extract of $10 C$. aeruginosa accessions were successfully detected using GC-MS analysis. Those phytochemicals were identified based on retention time, peak area, and molecular formula (Table 2). From ten different extracted C. aeruginosa accessions the identified compounds were categorized into different groups (Figure 1) i.e sesquiterpenes (36), monoterpenes (20), phenolics (5), diterpenes (4), phenanthrene (1), tetrapeptides (1), oxazole (1), triazine (1), piperidine (1), and oxygenated hydrocarbons (1). Details of each compound were presented in Table 2.

In detail, the ethanolic extract of $C$. aeruginosa accessions is mainly composed of sesquiterpenes (19.86 $43.72 \%)$ and monoterpenes $(6.3-25.86 \%)$. These findings correspond with research by Akarchariya et al. (2017), who reported the sesquiterpenes $(45.81 \%)$ and monoterpenes $(45.55 \%)$ as major components in essential oils of $C$. aeruginosa rhizome from Thailand. But, the monoterpenes were considered low than the earlier reported. The results found presented four compounds as dominant monoterpene metabolites namely eucalyptol (3.12-9.20\%), camphor (2.66-6.66\%), 1-sarvone oxide (2.15-9.33\%), and saussurea lactone (7.58-7.85\%). Meanwhile, the dominant sesquiterpenes are $\beta$-elemene $(1.16-2.67 \%)$, santonin (9.86-13.45\%), herbertenolide $(1.29-14.1 \%)$, epicurzerenone $(2.05$ $12.38 \%)$, isocurcumenol $(14.79-22.01 \%)$, germacrene B (1.82-3.68\%), $\alpha$-cadinene $(0.50-5.76 \%), \alpha$-guaiene $(0.08$ $2.54 \%), \alpha$-farnesene (1.26-2.96\%), 1,5,9,9-tetramethyl-2oxatricyclo[6.4.0.0(4,8)]dodecane $(14.17 \%)$, 4,8-dimethyl6 -phenylazulene (1.19-2.26\%), $\beta$-gurjunene (0.82-2.19\%), and lactarolide A $(2.05 \%)$. The most dominant metabolite in the ethanol extract of $C$. aeruginosa rhizome is isocurcumenol, with the highest production produced by $\mathrm{KP}$ accession. The isocurcumenol was detected in nine accessions except for $\mathrm{CB}$ accession.

However, the metabolites produced by all accessions studied are camphor and $\beta$-elements, which are monoterpenes and sesquiterpenes compounds, respectively. Several researchers have reported the results of profile metabolite content in $C$. aeruginosa rhizome in different extracts based on GC-MS analysis. The study from Akarchariya et al. (2017) reported that the major components of essential oils of $C$. aeruginosa rhizome from Thailand are camphor $(29.39 \%)$ and germacrone (21.21\%). The work from Kamazeri et al. (2012) showed that the essential oils of $C$. aeruginosa from Malaysia contain different major compounds identified as cycloisolongifolene, 8,9-dehydro formyl (35.29\%) and dihydrocostunolide $(22.51 \%)$. Meanwhile, different metabolites were identified by Simoh and Zainal (2015) in various extracts, such as methenolone (16.64\%), cycloisolongifolene, 8,9-dehydro9-formyl- (15.93\%), labd-13-en-15-oic acid,8,12-epoxy12-hydroxy- $\gamma$-lactone $(10.77 \%)$, propiolic acid, 3-(1hydroxy)-2 isopropyl-1,5-methylcyclohexyl) (7.84\%), 4oxo- $\beta$-isodamascol $(5.17 \%)$, velleral $(3.11 \%)$ and $Z-\alpha$ farnesene $(2.00 \%)$ in methyl tert-butyl ether extract, $\alpha-D$ glucopyranoside, 1,3,4,6 tetrakis-O-(TMS) (trimethylsilyl)$\beta$-D-fructofuranosyl 2,3,4,6-tetrakis-O-(TMS)- (38.08\%), d-glucose, 2,3,4,5,6-pentakis-O-(TMS)-, O-methyloxime (14.61\%), D-fructose, 1,3,4,5,6-pentakis-O-(TMS)-, Omethyloxime $(5.28 \%)$, isocitric acid (TMS) $(3.06 \%)$, oxalic acid, bis (TMS) ester (2.96\%), hexadecanoic acid, TMS ester $(2.16 \%)$, citric acid, ethyl ester, tri-TMS (1.91\%) and butanedioic acid, [(TMS) oxy]-, bis (TMS) ester (1.14\%) in methanol-chloroform extract, and cycloisolongifolene, 8, 9dehydro -9-formyl (15.70\%), propiolic acid, 3-(1-hydroxy2-isopropyl-5-methylcyclohexyl) (11.09\%), stearic acid, TMS ester $(2.78 \%)$, hexadecanoic acid, TMS ester $(2.33 \%)$, oleic acid, TMS ester (1.62\%), curzerene (1.56\%); Z- $\alpha$ farnesene $(1.52 \%)$, germacrone $(1.41 \%)$ and $\beta$-elemene $(1.33 \%)$ in chloroform extract. Therefore, it can be shown that, based on GC-MS examination, the form of extract and the source of the raw materials decide the quantity and quality of metabolites in the $C$. aeruginosa rhizome.

In this study, a total of 5 phenolics were also identified. Xanthotoxin, coumarin 138, trimethyl $(2,6$ ditert. butylphenoxy) silane, 4,5-dimethyl-1,2,3,6,7,8,8a,8boctahydrobiphenylene, and trans-longipinocarveol were detected. Xanthotoxin was highest detected in accession GK and MB but lowest identified in accession PK. Accession PW and MB contained coumarin 138. Meanwhile, 4,5-dimethyl-1,2,3,6,7,8,8a,8b-octahydrobiphenylene and trans-longipinocarveol compounds were detected in accessions GK and KL, respectively. Xanthotoxin shows antidepressant (Kowalczyk et al. 2021), anticancer (Mirzaei et al. 2017; Zhang et al. 2018), anti-inflammatory (Lee et al. 2017), and anticonvulsant (Zagaja et al. 2015, 2016) activities.

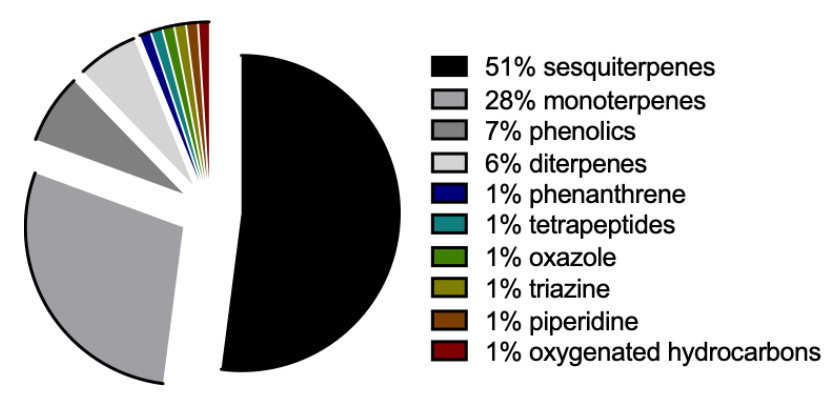

Figure 1. Pie Diagram displays the percentage of compound groups found in 10 C. aeruginosa accessions. 
Table 2. Volatile compounds identified from ethanol extract of $C$. aeruginosa rhizome accessions.

\begin{tabular}{|c|c|c|c|c|c|c|c|c|c|c|c|c|c|c|}
\hline \multirow[t]{2}{*}{ Compounds } & \multirow{2}{*}{$\begin{array}{l}\text { Group } \\
\text { compounds }\end{array}$} & \multirow[t]{2}{*}{ MF } & \multirow{2}{*}{\multicolumn{2}{|c|}{ MW RT }} & \multicolumn{10}{|c|}{ \% Total in accessions } \\
\hline & & & & & $\overline{\mathbf{K L}}$ & PK & $\mathbf{B H}$ & GK & KP & PW & MD & $\mathbf{L C}$ & MB & $\overline{C B}$ \\
\hline Xanthotoxin & Phenolics & $\mathrm{C}_{12} \mathrm{H}_{8} \mathrm{O}_{4}$ & 216 & 15.18 & & 8.66 & & 14.69 & & & & & 13.24 & \\
\hline Coumarin 138 & Phenolics & $\mathrm{C}_{14} \mathrm{H}_{15} \mathrm{NO}_{2}$ & 229 & 15.19 & & & & & & 17.66 & & & 1.2 & \\
\hline $\begin{array}{l}1,4,5,8,9,12- \\
\text { Hexahydrotriphenylene }\end{array}$ & Phenanthrene & $\mathrm{C}_{18} \mathrm{H}_{12} \mathrm{O}_{6}$ & 324 & 16.86 & 3.49 & & & & & & & & & \\
\hline $\begin{array}{l}\text { Trimethyl (2,6 ditert.- } \\
\text { butylphenoxy) silane }\end{array}$ & Phenolics & $\mathrm{C}_{17} \mathrm{H}_{30} \mathrm{OSi}$ & 279 & 16.99 & 1.33 & & & & & & & & & \\
\hline 6-Butyltetralin & Tetrapeptides & $\mathrm{C}_{14} \mathrm{H}_{20}$ & 188 & 17.88 & 5.54 & & & & & & & & & \\
\hline $\begin{array}{l}\text { 4,5-Dimethyl-1,2,3,6,7,8,8a,8b- } \\
\text { octahydrobiphenylene }\end{array}$ & Phenolics & $\mathrm{C}_{14} \mathrm{H}_{20}$ & 188 & 17.89 & & & & 3.72 & & & & & & \\
\hline Valerenol & Sesquiterpenes & $\mathrm{C}_{15} \mathrm{H}_{24} \mathrm{O}$ & 220 & 18.70 & & 2.4 & & & & & & & & \\
\hline trans-Longipinocarveol & Phenolics & $\mathrm{C}_{15} \mathrm{H}_{24} \mathrm{O}$ & 220 & 18.70 & 1.93 & & & & & & & & & \\
\hline 4-Phenylthioacetophenone & Oxazole & $\mathrm{C}_{14} \mathrm{H}_{12} \mathrm{OS}$ & 228 & 19.50 & 0.41 & & & & & & & & & \\
\hline Cymetrin & Triazine & $\mathrm{C}_{8} \mathrm{H}_{15} \mathrm{~N}_{5} \mathrm{~S}$ & 213 & 19.50 & & 0.55 & & & & & & & & \\
\hline 2-Piperidone & Piperidine & $\mathrm{C}_{5} \mathrm{H}_{9} \mathrm{NO}$ & 99 & 19.90 & & 0.61 & & 0.18 & & & & & & \\
\hline 2-Nonadecanone & $\begin{array}{l}\text { Oxygenated } \\
\text { hydrocarbons }\end{array}$ & $\mathrm{C}_{19} \mathrm{H}_{38} \mathrm{O}$ & 282 & 20.32 & & & & & & & & & & 0.36 \\
\hline (+)-Camphene & Monoterpenes & $\mathrm{C}_{10} \mathrm{H}_{16}$ & 136 & 6.29 & & & & & & & & & & 0.09 \\
\hline$\beta$-Pinene & Monoterpenes & $\mathrm{C}_{10} \mathrm{H}_{16}$ & 136 & 6.69 & & & & 0.22 & & & & & & 0.08 \\
\hline Eucalyptol & Monoterpenes & $\mathrm{C}_{10} \mathrm{H}_{18} \mathrm{O}$ & 154 & 7.47 & 3.71 & 3.56 & 3.87 & & 4.69 & 5.43 & 9.2 & 3.49 & 3.79 & 3.12 \\
\hline Camphor & Monoterpenes & $\mathrm{C}_{10} \mathrm{H}_{16} \mathrm{O}$ & 152 & 9.05 & 3.19 & 3.32 & 3.61 & 3.58 & 3.84 & 4.3 & 6.66 & 2.66 & 3.14 & 2.64 \\
\hline Camphene & Monoterpenes & $\mathrm{C}_{10} \mathrm{H}_{16}$ & 136 & 9.37 & 0.71 & & & 0.86 & & & & & 0.7 & \\
\hline Isononyl acrylate & Monoterpenes & $\mathrm{C}_{13} \mathrm{H}_{20} \mathrm{O}_{2}$ & 208 & 9.37 & & & & & & 1.02 & & & & \\
\hline$\alpha$-Pinene & Monoterpenes & $\mathrm{C}_{10} \mathrm{H}_{16}$ & 136 & 9.80 & & 0.37 & & & & & & & & \\
\hline $\begin{array}{l}\text { Cyclopropane, 1-(1- } \\
\text { methylethenyl)-2-(2-methyl-1- } \\
\text { propenyl)-, (1R-trans)- }\end{array}$ & Monoterpenes & $\mathrm{C}_{10} \mathrm{H}_{16}$ & 136 & 9.80 & & & & & & & & & 0.32 & \\
\hline Terpineol & Monoterpenes & $\mathrm{C}_{10} \mathrm{H}_{18} \mathrm{O}$ & 154 & 9.81 & & & & 0.5 & & & & & & \\
\hline Carvone & Monoterpenes & $\mathrm{C}_{10} \mathrm{H}_{14} \mathrm{O}$ & 150 & 10.42 & 0.12 & & & & & & & & & 0.09 \\
\hline 2-Carene & Monoterpenes & $\mathrm{C}_{10} \mathrm{H}_{16}$ & 136 & 12.09 & & & & & & & & & & 0.09 \\
\hline 4-Carene & Monoterpenes & $\mathrm{C}_{10} \mathrm{H}_{16}$ & 136 & 12.09 & & 0.09 & & & & & & & & \\
\hline Isoterpinolene & Monoterpenes & $\mathrm{C}_{10} \mathrm{H}_{16}$ & 136 & 12.10 & 0.11 & & & & & & & & & \\
\hline$\alpha$-Terpinene & Monoterpenes & $\mathrm{C}_{10} \mathrm{H}_{16}$ & 136 & 12.12 & & & & 0.26 & & & & & & \\
\hline (+)-gamma-cadinene & Monoterpenes & $\mathrm{C}_{15} \mathrm{H}_{24}$ & 204 & 13.88 & & & & & & & & & & 0.19 \\
\hline$\beta$-Ionene & Monoterpenes & $\mathrm{C}_{13} \mathrm{H}_{20} \mathrm{O}$ & 192 & 16.32 & & & & & & & & 1.69 & 0.09 & \\
\hline 1-Carvone oxide & Monoterpenes & $\mathrm{C}_{10} \mathrm{H}_{14} \mathrm{O}_{2}$ & 166 & 17.17 & 7.54 & 3.34 & 9.33 & 5.45 & 2.5 & 2.37 & 2.15 & & & \\
\hline Saussurea lactone & Monoterpenes & $\mathrm{C}_{15} \mathrm{H}_{22} \mathrm{O}_{2}$ & 234 & 17.17 & & 7.58 & & & & & 7.85 & & & \\
\hline $1,1,3,4,4,6$-Hexamethyltetralin & Monoterpenes & $\mathrm{C}_{16} \mathrm{H}_{24}$ & 216 & 18.45 & & & & 0.88 & & & & & & \\
\hline $\begin{array}{l}\text { 2-Isopropylidene-3-methylhexa- } \\
\text { 3,5-dienal }\end{array}$ & Monoterpenes & $\mathrm{C}_{10} \mathrm{H}_{14} \mathrm{O}$ & 150 & 18.49 & & & & & & & & 0.98 & & \\
\hline$\delta$-Elemene & Sesquiterpenes & $\mathrm{C}_{15} \mathrm{H}_{24}$ & 204 & 12.11 & & & & & & & & & 0.17 & \\
\hline$\beta$-Elemene & Sesquiterpenes & $\mathrm{C}_{15} \mathrm{H}_{24}$ & 204 & 12.80 & 1.51 & 1.46 & 1.75 & 1.86 & 1.72 & 2.67 & 2.36 & 1.2 & 2.14 & 1.16 \\
\hline Caryophyllene & Sesquiterpenes & $\mathrm{C}_{15} \mathrm{H}_{24}$ & 204 & 13.24 & & & & & & & & & & 0.13 \\
\hline (E)- $\beta$-Farnesene & Sesquiterpenes & $\mathrm{C}_{15} \mathrm{H}_{24}$ & 204 & 13.50 & & & & & & & & & & 0.29 \\
\hline$+\alpha$-Amorphene & Sesquiterpenes & $\mathrm{C}_{15} \mathrm{H}_{24}$ & 204 & 13.87 & 0.22 & & & & & & & & & \\
\hline Epizonarene & Sesquiterpenes & $\mathrm{C}_{15} \mathrm{H}_{24}$ & 204 & 13.87 & & & & 0.33 & & & & & & \\
\hline Cyclo- $\gamma$-Cadinene & Sesquiterpenes & $\mathrm{C}_{15} \mathrm{H}_{24}$ & 204 & 13.87 & & 0.2 & & 0.27 & & & & & & \\
\hline Aromadendrene & Sesquiterpenes & $\mathrm{C}_{15} \mathrm{H}_{24}$ & 204 & 14.08 & & & & & & & & 1.57 & & \\
\hline Cyclosativene & Sesquiterpenes & $\mathrm{C}_{15} \mathrm{H}_{24}$ & 204 & 14.08 & 1.33 & & & & & & & & & \\
\hline Allo-Aromadendrene & Sesquiterpenes & $\mathrm{C}_{15} \mathrm{H}_{24}$ & 204 & 14.08 & & & & & 2 & & & & & \\
\hline$\beta$-Selinene & Sesquiterpenes & $\mathrm{C}_{15} \mathrm{H}_{24}$ & 204 & 14.08 & & & 1.6 & 1.24 & & 1.52 & 1.97 & & & 1.27 \\
\hline$\beta$-Guaiene & Sesquiterpenes & $\mathrm{C}_{15} \mathrm{H}_{24}$ & 204 & 14.08 & & & & & & & & & 1.21 & \\
\hline$\alpha$-Selinene & Sesquiterpenes & $\mathrm{C}_{15} \mathrm{H}_{24}$ & 204 & 14.17 & & & & 0.61 & & & & & 0.22 & \\
\hline$\delta$-Cedrol & Sesquiterpenes & $\mathrm{C}_{15} \mathrm{H}_{26} \mathrm{O}$ & 222 & 14.35 & & & & & & & & & & 0.21 \\
\hline$\alpha$-Amorphene & Sesquiterpenes & $\mathrm{C}_{15} \mathrm{H}_{24}$ & 204 & 14.35 & & 0.21 & & & & & & & & \\
\hline$\alpha$-Copaene & Sesquiterpenes & $\mathrm{C}_{15} \mathrm{H}_{24}$ & 204 & 14.35 & & & & & & & & & 0.22 & \\
\hline$\alpha$-Calacorene & Sesquiterpenes & $\mathrm{C}_{15} \mathrm{H}_{20}$ & 200 & 14.60 & 0.15 & 0.15 & & & & & & & & \\
\hline$\beta$-Caryophyllene & Sesquiterpenes & $\mathrm{C}_{15} \mathrm{H}_{24}$ & 204 & 14.76 & & & & & & & & & 0.58 & \\
\hline Santonin & Sesquiterpenes & $\mathrm{C}_{15} \mathrm{H}_{18} \mathrm{O}_{3}$ & 246 & 15.19 & & & & & & & & 11.21 & 13.45 & 9.86 \\
\hline Herbertenolide & Sesquiterpenes & $\mathrm{C}_{15} \mathrm{H}_{18} \mathrm{O}_{2}$ & 230 & 15.19 & & & & & 14.1 & & & & 1.29 & \\
\hline Epicurzerenone & Sesquiterpenes & $\mathrm{C}_{15} \mathrm{H}_{18} \mathrm{O}$ & 230 & 15.20 & 12.38 & 2.24 & & 3.09 & 2.42 & 2.05 & 13.4 & & 7.01 & 2.18 \\
\hline Isocurcumenol & Sesquiterpenes & $\mathrm{C}_{15} \mathrm{H}_{22} \mathrm{O}_{2}$ & 234 & 15.63 & 14.79 & 15.44 & 21 & 17.08 & 22.01 & 19.72 & 21.12 & 17.49 & 5.06 & \\
\hline Germacrene B & Sesquiterpenes & $\mathrm{C}_{15} \mathrm{H}_{24}$ & 204 & 15.90 & 3.68 & & & & & & & & 1.82 & \\
\hline$\alpha$-Cadinene & Sesquiterpenes & $\mathrm{C}_{15} \mathrm{H}_{24}$ & 204 & 15.94 & & & & & & & & 5.76 & 0.5 & 1.04 \\
\hline
\end{tabular}




\begin{tabular}{|c|c|c|c|c|c|c|c|c|c|c|}
\hline Caryophyllene & Sesquiterpenes & $\mathrm{C}_{15} \mathrm{H}_{24}$ & 204 & 16.09 & 1.06 & & & & & 0.84 \\
\hline Ishwarane & Sesquiterpenes & $\mathrm{C}_{15} \mathrm{H}_{24}$ & 204 & 16.38 & & & 1.74 & & & 0.64 \\
\hline$\alpha$-Guaiene & Sesquiterpenes & $\mathrm{C}_{15} \mathrm{H}_{24}$ & 204 & 16.40 & & & & 2.54 & & 0.08 \\
\hline$\alpha$-Farnesene & Sesquiterpenes & $\mathrm{C}_{15} \mathrm{H}_{24}$ & 204 & 16.50 & 1.34 & 1.26 & & & 2.96 & \\
\hline $\begin{array}{l}\text { 1,5,9,9-Tetramethyl-2- } \\
\text { oxatricyclo[6.4.0.0(4,8)]dodecane }\end{array}$ & Sesquiterpenes & $\mathrm{C}_{15} \mathrm{H}_{26} \mathrm{O}$ & 222 & 16.76 & & & 14.17 & & & \\
\hline 4,8-Dimethyl-6-phenylazulene & Sesquiterpenes & $\mathrm{C}_{18} \mathrm{H}_{16}$ & 232 & 17.38 & & & & 1.19 & & \\
\hline$\beta$-Ionene & Sesquiterpenes & $\mathrm{C}_{13} \mathrm{H}_{18}$ & 174 & 18.03 & & & & & & \\
\hline$\beta$-Gurjunene & Sesquiterpenes & $\mathrm{C}_{14} \mathrm{H}_{20}$ & 188 & 18.24 & & & 0.82 & & 2.19 & \\
\hline $\begin{array}{l}\text { 1,10: 4,5-Diepoxy-7(11)- } \\
\text { germacren-8-one }\end{array}$ & Sesquiterpenes & $\mathrm{C}_{15} \mathrm{H}_{22} \mathrm{O}_{3}$ & 250 & 18.70 & & & 1.41 & & & \\
\hline Lactarolide A & Sesquiterpenes & $\mathrm{C}_{15} \mathrm{H}_{22} \mathrm{O}_{5}$ & 282 & 19.18 & & & 2.05 & & & \\
\hline Versalide & Sesquiterpenes & $\mathrm{C}_{18} \mathrm{H}_{26} \mathrm{O}$ & 258 & 19.50 & & & & & & \\
\hline Xeniaphyllenol B & Diterpenes & $\mathrm{C}_{20} \mathrm{H}_{32} \mathrm{O}_{2}$ & 305 & 11.33 & & & 0.17 & & & \\
\hline $\begin{array}{l}\text { (10-[(Acetyloxy) methyl]-9- } \\
\text { antrhryl) methyl acetate }\end{array}$ & Diterpenes & $\mathrm{C}_{20} \mathrm{H}_{18} \mathrm{O}_{4}$ & 322 & 15.90 & & 3.64 & & & & 1.52 \\
\hline Columbin & Diterpenes & $\mathrm{C}_{20} \mathrm{H}_{22} \mathrm{O}_{6}$ & 358 & 22.63 & & & 0.96 & & & \\
\hline Labd-7,13-(E)-dien-15-ol & Diterpenes & $\mathrm{C}_{20} \mathrm{H}_{34} \mathrm{O}$ & 290 & 22.65 & & & 1.95 & & & \\
\hline
\end{tabular}

Note: MF: molecule formula; MW: molecule weight (g/mol); and RT: retention time (min)

Regarding diterpenes compound, a total of 4 compounds were identified. Xeniaphyllenol B, (10[(acetyloxy) methyl]-9-antrhryl) methyl acetate, columbin, and labd-7,13-(E)-dien-15-ol were detected. The distribution between accessions of these compounds showed that the accessions GK contained xeniaphyllenol B, accession PK and MB contained (10-[(acetyloxy) methyl]9-antrhryl) methyl acetate, accession GK contained columbin, and accession BH contained labd-7,13-(E)-dien15-ol. Columbin is known to possess anticancer (Kohno et al. 2002) and anti-inflammatory (Ibrahim Abdelwahab et al. 2012) effects.

The following six other group compounds namely phenanthrene $(1,4,5,8,9,12$-hexahydrotriphenylene), tetrapeptides (6-butyltetralin), oxazole (4-phenylthioacetophenone), triazine (cymetrin), piperidine (2-piperidone), and oxygenated hydrocarbons (2-nonadecanone) were also detected in accessions studied (Table 2). Accession KL contained 1,4,5,8,9,12-hexahydrotriphenylene, 6butyltetralin, and 4-phenylthioacetophenone compounds. Cymetrin and 2- nonadecanone were recorded in accession $\mathrm{PK}$ and $\mathrm{CB}$, respectively. 2-Piperidone was detected in two accessions (PK and GK).

\section{Anticancer activity}

Cytotoxic activity in ethanol extract of $10 \mathrm{C}$. aeruginosa accessions against MCF-7 and Vero cell lines is presented in Figure 2. In the Vero as a normal cell, the accession PW showed maximum cytotoxic activity with a value of $45.17 \%$. This value not significantly different from doxorubicin (45.93\%), accession KP (44.89\%), GK (39.74\%), and KL (37.64\%). In comparison, accession MD presented the lowest cytotoxic activity with a value of $15.13 \%$. Meanwhile, in the MCF-7, the cytotoxic activity ranged between $3.04 \%$ (accession $\mathrm{CB}$ ) to $53.65 \%$ (accession MD). All accessions studied showed significantly different from doxorubicin $(65.05 \%)$ at $\mathrm{p} \leq$ 0.05 . A successful anticancer is that it can destroy cancer cells, but must have a limited impact on normal growth of cells (Safarzadeh et al. 2014). Results suggested selecting the accession MD to continue developing $C$. aeruginosa varieties with high anticancer activity through a breeding program. Atun et al. (2010) reported that methanol extract and hexane and chloroform fractions of $C$. aeruginosa have potent anticancer activity against the MCF-7 and $\mathrm{Ca}$ Ski cell lines. However, recently no study is found on ethanolic extract of $C$. aeruginosa for anticancer potential against the MCF-7 cell. Consequently, the results of this study have shown that the ethanolic extracts of $C$. aeruginosa can be produced as drug molecules for cancer disease.
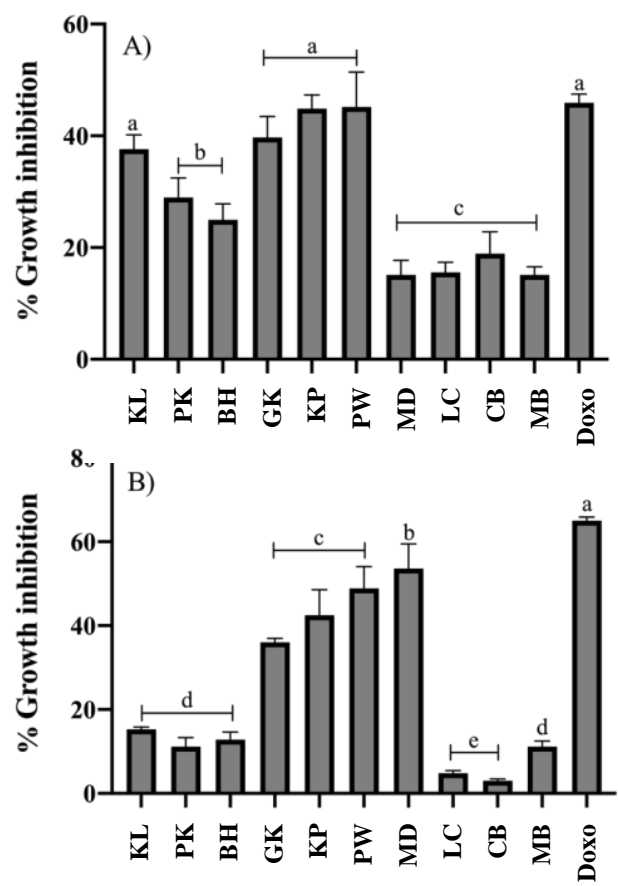

Figure 2. Antiproliferative activity of the ethanol extract from the rhizome of Curcuma aeruginosa accessions against (A) Vero and (B) MCF-7 cell lines. Doxo, doxorubicin as a positive control. Values show the mean \pm SD for $n=3$. According to the ScottKnott test at $\mathrm{p} \leq 0.05$, two independent studies (A and B) with different superscripts (a, b, c, d, e) differ significantly. 


\section{Multivariate analysis}

In this work, the principal component analysis (PCA) and hierarchical cluster analysis (HCA)-heatmap dendrogram in $C$. aeruginosa studied accessions were used for multivariate analysis, namely chemometric. The chemometric allows for the quantification and enhancement of the understanding of metabolites information and the association of quality traits to analytical instruments data (Batubara et al. 2020). It has been used to determine the chemical components in plants that contribute to its medicinal benefits (Abbasi et al. 2018; Guo et al. 2020; Windarsih et al. 2019).

PCA analysis revealed that the first two components (shown in Figure 3) accounted for a total of $35.2 \%$ of the data variance explained. The individual score plot (Figure 3A) and loading plot (Figure 3B) represent the significant differences between the accessions studied. The diversity of data explained by PCA is low, so HCA analysis is often required to better describe the data (Khumaida et al. 2019; Péroumal et al. 2017). In chemometric analysis (PCA and HCA), it was resulted five clusters. Cluster 1 consisted of one accession viz. $\mathrm{CB}$ due to their high metabolite content of 4,8-dimethyl-6-phenylazulene (M62), (E)- $\beta$-farnesene
(M36), $\delta$-cedrol (M46), versalide (M67), (+)-gammacadinene (M27), 2-carene (M23), 2-nonadecanone (M12), (+)-camphene (M13), caryophyllene (M35), $\beta$-ionene (M63), and santonin (M51). Cluster 2 was composed one accession: KL. This accession presented the highest carvone (M22), 1,4,5,8,9,12-hexahydrotriphenylene (M3), isoterpinolene (M25), cyclosativene (M41), translongipinocarveol (M8), trimethyl (2,6 ditert.-butylphenoxy) silane (M4), 6-butyltetralin (M5), 4phenylthioacetophenone (M9), $+\alpha$-amorphene (M37), epicurzerenone (M53), camphene (M17), germacrene B (M55), and caryophyllene (M57) metabolites. Accession GK grouped in cluster 3. This group was characterized by high $\beta$-pinene (M14), $\alpha$-selinene (M45), 4,5-dimethyl1,2,3,6,7,8,8a,8b-octahydrobiphenylene (M6), epizonarene (M38), terpineol (M21), Columbin (M70), xeniaphyllenol B (M68), $\alpha$-terpinene (M26), 1,1,3,4,4,6-hexamethyltetralin (M31), xanthotoxin (M1), and cyclo- $\gamma$-cadinene (M39). Cluster 4 consisted accession PK with characterized by high $\alpha$-calacorene (M49), (10-[(acetyloxy) methyl]-9antrhryl) methyl acetate (M69), 2-piperidone (M11), cymetrin (M10), 4-carene (M24), $\alpha$-amorphene (M47), valerenol (M7), and $\alpha$-pinene (M19) metabolites.
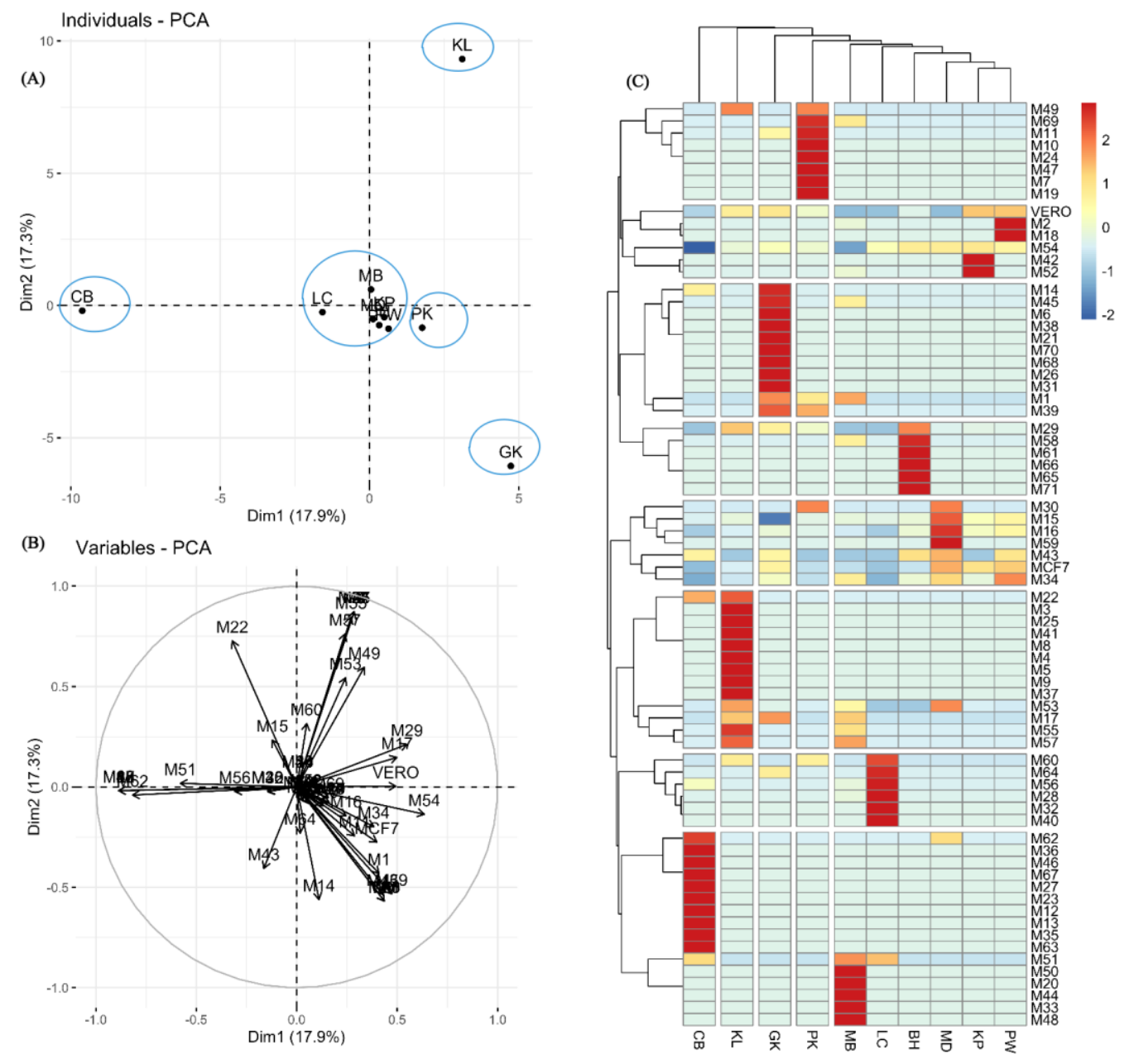

Figure 3. Score plot (A), loading plot (B) and HCA-heatmap dendrogram (C) of PCA for C. aeruginosa accessions using the metabolites (M1 - M71, see in Table 2) and cytotoxicity activities against Vero and MCF-7 cell lines matrix as input variables. The darker red, yellow and darker blue presented higher, moderate and lower metabolites contents and cytotoxic activities, respectively. 
Cluster 5 represented $\mathrm{MB}, \mathrm{LC}, \mathrm{BH}, \mathrm{MD}, \mathrm{KP}$, and $\mathrm{PW}$ accessions. Interestingly, accessions studied in cluster 5 were associated with metabolites and cytotoxic activities. The highest cytotoxic activities against MCF-7 cell line found in accession MD (53.65\%) followed with accession PW (48.91\%) and KP (42.52\%), but the accessions PW $(45.17 \%)$ and KP $(44.89 \%)$ also high cytotoxic activity against Vero cell line. These accessions had high metabolites saussurea lactone (M30), eucalyptol (M15), camphor (M16), epicurzerenone (M53), and $\beta$-elemene (M34) and cytotoxic activity against the MCF-7 cell line, indicating the association of maximum metabolites with anticancer activity. Past research has shown these compounds from several medicinal plants to be useful as anticancer. Saussurea lactone was isolated from the roots of Saussurea lappa, which exhibited potent anticancer properties (Robinson et al. 2008). Yang et al. (2010) showed that the eucalyptol compound from the essential oil of Amomum tsao-ko had anticancer activity against HepG2 carcinoma cell line. The camphor compound found in the essential oil of Origanum vulgare has been shown to have anti-cancer activity (Elansary et al. 2018). Rahman et al. (2013) isolated epicurzerenone (curzerenone as synonym name) from Curcuma zedoaria, which exhibited cytotoxicity on Ca Ski, MCF-7, and HCT-116 cancer cell lines. Meanwhile, $\beta$-elemene has been reported to have anticancer activity for several types of cancer (Deng et al. 2020; Zhai et al. 2019). The future will combine the main target of new varieties development for $C$. aeruginosa as anticancer sources high metabolite content and anticancer activity. The results indicated that phytochemical parameters including saussurea lactone, eucalyptol, camphor, epicurzerenone, and $\beta$-elemene could be used as a selection parameter the development $C$. aeruginosa varieties in addition to anticancer activity through in the breeding program.

The results show that the ethanolic extract of $C$. aeruginosa accessions contains a variable pattern of sesquiterpenes, monoterpenes, phenolics, diterpenes, and others. Isocurcumenol was found as major compound of accessions studied. Camphor and $\beta$-elemene compounds were detected in majority of accessions studied. The chemometric analysis concluded that the saussurea lactone, eucalyptol, camphor, epicurzerenone, and $\beta$-elemene metabolites are responsible for anticancer activity against the MCF-7 cell line in ethanolic extract of $C$. aeruginosa accessions. Accession MD had the maximum anticancer activity; therefore, it can select this accession to continue developing $C$. aeruginosa varieties through the breeding program as a source of drug metabolites producing to treat cancer diseases.

\section{ACKNOWLEDGEMENTS}

This study was supported by the PTUPT research grant of IPB University (1714/IT3.11/PN/2018) from the Ministry of Research, Technology and Higher Education of the Republic of Indonesia. Thank you to Topik Ridwan for identifying the plant material used in this paper.

\section{REFERENCES}

Abbasi S, Benvidi A, Gharaghani S, Rezaeinasab M. 2018. Chemometric studies of thymol binding with bovine serum albumin: A developing strategy for the successful investigation of pharmacological activity. Bioelectrochemistry 124: 172-184. DOI: 10.1016/j.bioelechem.2018.07.017.

Akarchariya N, Sirilun S, Julsrigival J, Chansakaowa S. 2017. Chemical profiling and antimicrobial activity of essential oil from Curcuma aeruginosa Roxb., Curcuma glans K. Larsen \& J. Mood and Curcuma cf. xanthorrhiza Roxb. collected in Thailand. Asian Pac J Trop Biomed 7: 881-885. DOI: 10.1016/j.apjtb.2017.09.009.

Atun S, Aznam N, Arianingrum R, Nurestri S. 2010. Cytotoxic effects of plant growth extracts temu giring (Curcuma heyneana) and temu ireng (Curcuma aeruginosa) against several cancer cells. J Penelit Saintek 15: 1-9

Batubara I, Komariah K, Sandrawati A, Nurcholis W. 2020. Genotype selection for phytochemical content and pharmacological activities in ethanol extracts of fifteen types of Orthosiphon aristatus (Blume) Miq. leaves using chemometric analysis. Sci Rep 10: 20945. DOI: 10.1038/s41598-020-77991-2.

Deng M, Liu B, Song H, Yu R, Zou D, Chen Y, et al. 2020. $\beta$-Elemene inhibits the metastasis of multidrug-resistant gastric cancer cells through miR-1323/Cbl-b/EGFR pathway. Phytomedicine 69: 153184. DOI: $10.1016 /$ j.phymed.2020.153184.

Elansary HO, Abdelgaleil SAM, Mahmoud EA, Yessoufou K, Elhindi K, El-Hendawy S. 2018. Effective antioxidant, antimicrobial and anticancer activities of essential oils of horticultural aromatic crops in northern Egypt. BMC Compl Altern Med 18: 214. DOI: 10.1186/s12906-018-2262-1.

Fitria R, Seno DSH, Priosoeryanto BP, Nurcholis W. 2019. Volatile compound profiles and cytotoxicity in essential oils from rhizome of Curcuma aeruginosa and Curcuma zanthorrhiza. Biodiversitas 20: 2943-2948. DOI: 10.13057/biodiv/d201024.

Ghimire BK, Yoo JH, Yu CY, Chung I-M. 2017. GC-MS analysis of volatile compounds of Perilla frutescens Britton var. Japonica accessions: Morphological and seasonal variability. Asian Pac J Trop Med 10: 643-651. DOI: 10.1016/j.apjtm.2017.07.004.

Guo L, Gong M, Wu S, Qiu F, Ma L. 2020. Identification and quantification of the quality markers and anti-migraine active components in Chuanxiong Rhizoma and Cyperi Rhizoma herbal pair based on chemometric analysis between chemical constituents and pharmacological effects. J Ethnopharmacol 246: 112228. DOI: 10.1016/j.jep.2019.112228.

Hossain CF, Al-Amin M, Sayem ASM, Siragee IH, Tunan AM, Hassan F, et al. 2015. Antinociceptive principle from Curcuma aeruginosa. BMC Compl Altern Med 15: 191 DOI: 10.1186/s12906-015-0720-6.

Ibrahim Abdelwahab S, Syaed Koko W, Mohamed Elhassan Taha M, Mohan S, Achoui M, Ameen Abdulla M, et al. 2012. In vitro and in vivo anti-inflammatory activities of columbin through the inhibition of cycloxygenase- 2 and nitric oxide but not the suppression of NF- $\kappa$ B translocation. Eur J Pharmacol 678: 61-70 DOI: 10.1016/j.ejphar.2011.12.024.

Kamazeri TSAT, Samah OA, Taher M, Susanti D, Qaralleh H. 2012. Antimicrobial activity and essential oils of Curcuma aeruginosa, Curcuma mangga, and Zingiber cassumunar from Malaysia. Asian Pac J Trop Med 5: 202-209. DOI: 10.1016/S1995-7645(12)60025-X.

Khumaida N, Syukur M, Bintang M, Nurcholis W. 2019. Phenolic and flavonoid content in ethanol extract and agro-morphological diversity of Curcuma aeruginosa accessions growing in west java, Indonesia. Biodiversitas 20: 656-663. DOI: 10.13057/biodiv/d200306.

Kohno H, Maeda M, Tanino M, Tsukio Y, Ueda N, Wada K, et al. 2002. A bitter diterpenoid furanolactone columbin from Calumbae Radix inhibits azoxymethane-induced rat colon carcinogenesis. Cancer Lett 183: 131-139. DOI: 10.1016/S0304-3835(02)00159-3.

Kowalczyk J, Nakos-Bimpos M, Polissidis A, Dalla C, Kokras N, Skalicka-Wozniak K, et al. 2021. Xanthotoxin affects depressionrelated behavior and neurotransmitters content in a sex-dependent manner in mice. Behav Brain Res 399: 112985. DOI: 10.1016/j.bbr.2020.112985. 
Lee S-B, Lee WS, Shin J-S, Jang DS, Lee KT. 2017. Xanthotoxin suppresses LPS-induced expression of iNOS, COX-2, TNF- $\alpha$, and IL6 via AP-1, NF-KB, and JAK-STAT inactivation in RAW 264.7 macrophages. Int Immunopharmacol 49: 21-29. DOI: 10.1016/j.intimp.2017.05.021.

Mahajan M, Kuiry R, Pal PK. 2020. Understanding the consequence of environmental stress for accumulation of secondary metabolites in medicinal and aromatic plants. J Appl Res Med Aromat Plants 18: 100255 DOI: $10.1016 /$ j.jarmap.2020.100255.

Mirzaei SA, Gholamian Dehkordi N, Ghamghami M, Amiri AH, Dalir Abdolahinia E, Elahian F. 2017. ABC-transporter blockage mediated by xanthotoxin and bergapten is the major pathway for chemosensitization of multidrug-resistant cancer cells. Toxicol Appl Pharmacol 337: 22-29. DOI: 10.1016/j.taap.2017.10.018.

Moektiwardoyo WM, Tjitraresmi A, Susilawati Y, Iskandar Y, Halimah E, Zahryanti D. 2014. The potential of dewa leaves (Gynura pseudochina (L) D.C) and temu ireng rhizomes (Curcuma aeruginosa Roxb.) as medicinal herbs for dengue fever treatment. Procedia Chem 13: 134-141. DOI: 10.1016/j.proche.2014.12.017.

Ncube B, Finnie JF, Van Staden J. 2012. Quality from the field: The impact of environmental factors as quality determinants in medicinal plants. South African J Bot 82: 11-20. DOI: 10.1016/j.sajb.2012.05.009.

Nurcholis W, Khumaida N, Syukur M, Bintang M. 2017. Evaluation of free radical scavenging activity in ethanolic extract from promising accessions of Curcuma aeruginosa RoxB. Molekul 12: 133-138.

Nurcholis W, Khumaida N, Syukur M, Bintang M. 2019. Variability of curcumin, demethoxycurcumin and bisdemethoxycurcumin contents in ethanolic extract from ten Curcuma aeruginosa Roxb. cultivated in West Java, Indonesia. Asian $\mathbf{J}$ Chem 31. DOI 10.14233/ajchem.2019.22128.

Nurcholis W, Khumaida N, Syukur M, Bintang M. 2016a. Variability of curcuminoid content and lack of correlation with cytotoxicity in ethanolic extracts from 20 accessions of Curcuma aeruginosa RoxB. Asian Pacific J Trop Dis 6: 887-891. DOI: 10.1016/S22221808(16)61152-0

Nurcholis W, Khumaida N, Syukur M, Bintang M. 2016b. Variability of total phenolic and flavonoid content and antioxidant activity among 20 Curcuma aeruginosa Roxb. accessions of Indonesia. Asian J Biochem 11: 142-148. DOI: 10.3923/ajb.2016.142.148.

Nurcholis W, Khumaida N, Syukur M, Bintang M, Ardyani IDAA. 2015. Phytochemical screening, antioxidant and cytotoxic activities in extracts of different rhizome parts from Curcuma aeruginosa Roxb. Intl J Res Ayurveda Pharm 6: 634-637. DOI: 10.7897/22774343.065118.

Péroumal A, Adenet S, Rochefort K, Fahrasmane L, Aurore G. 2017. Variability of traits and bioactive compounds in the fruit and pulp of six mamey apple (Mammea americana L.) accessions. Food Chem 234: 269-275. DOI: 10.1016/j.foodchem.2017.04.145.

Pumthong G, Asawanonda P, Varothai S, Jariyasethavong V, Triwongwaranat D, Suthipinittharm P, et al. 2012. Curcuma aeruginosa, a novel botanically derived $5 \alpha$-reductase inhibitor in the treatment of male-pattern baldness: a multicenter, randomized, double-blind, placebo-controlled study. J Dermatolog Treat 23: 385392. DOI: $10.3109 / 09546634.2011 .568470$.

Rahman SNSA, Wahab NA, Malek SNA. 2013. In vitro morphological assessment of apoptosis induced by antiproliferative constituents from the rhizomes of Curcuma zedoaria. Evid Based Compl Alternat Med 2013: 257108. DOI: $10.1155 / 2013 / 257108$.

Reanmongkol W, Subhadhirasakul S, Khaisombat N, Fuengnawakit P, Jantasila S, Khamjun A. 2006. Investigation the antinociceptive, antipyretic and anti-inflammatory activities of Curcuma aeruginosa Roxb. extracts in experimental animals. Songklanakarin J Sci Technol 28: $999-1008$
Robinson A, Kumar TV, Sreedhar E, Naidu VGM, Krishna SR, Babu KS, et al. 2008. A new sesquiterpene lactone from the roots of Saussurea lappa: Structure-anticancer activity study. Bioorg Med Chem Lett 18: 4015-4017 DOI: 10.1016/j.bmcl.2008.06.008.

Safarzadeh E, Sandoghchian Shotorbani S, Baradaran B. 2014. Herbal medicine as inducers of apoptosis in cancer treatment. Adv Pharm Bull 4: 421-427. DOI: 10.5681/apb.2014.062.

Simoh S, Zainal A. 2015. Chemical profiling of Curcuma aeruginosa Roxb. rhizome using different techniques of solvent extraction. Asian Pac J Trop Biomed 5: 412-417. DOI: 10.1016/S2221-1691(15)303786.

Sirirugsa P, Larsen K, Maknoi C. 2007. The genus Curcuma L. (Zingiberaceae): distribution and classification with reference to species diversity in Thailand. Gard Bull Sing 59: 203-220.

Srivilai J, Waranuch N, Tangsumranjit A, Khorana N, Ingkaninan K. 2018. Germacrone and sesquiterpene-enriched extracts from Curcuma aeruginosa Roxb. increase skin penetration of minoxidil, a hair growth promoter. Drug Deliv Transl Res 8: 140-149. DOI: 10.1007/s13346-017-0447-7.

Suphrom N, Pumthong G, Khorana N, Waranuch N, Limpeanchob N, Ingkaninan K. 2012. Anti-androgenic effect of sesquiterpenes isolated from the rhizomes of Curcuma aeruginosa Roxb. Fitoterapia 83: 864871. DOI: $10.1016 /$ j.fitote.2012.03.017.

Thaina $\mathrm{P}$, Tungcharoen $\mathrm{P}$, Wongnawa $\mathrm{M}$, Reanmongkol W, Subhadhirasakul S. 2009. Uterine relaxant effects of Curcuma aeruginosa Roxb. rhizome extracts. J Ethnopharmacol 121: 433-443. DOI: $10.1016 /$ j.jep.2008.10.022

Theanphong O, Mingvanish W, Kirdmanee C. 2015. Chemical constituents and biological activities of essential oil from Curcuma aeruginosa Roxb. rhizome. Bull Heal Sci Technol 13: 6-16.

Ukwubile CA, Ahmed A, Katsayal UA, Ya'u J, Mejida S. 2019. GC-MS analysis of bioactive compounds from Melastomastrum capitatum (Vahl) Fern. leaf methanol extract: An anticancer plant. Sci African 3: e00059. DOI: 10.1016/j.sciaf.2019.e00059.

Vijayarathna S, Sasidharan S. 2012. Cytotoxicity of methanol extracts of Elaeis guineensis on MCF-7 and Vero cell lines. Asian Pac J Trop Biomed 2: 826-829. DOI: 10.1016/S2221-1691(12)60237-8.

Windarsih A, Rohman A, Swasono RT. 2019. Application of 1H-NMR based metabolite fingerprinting and chemometrics for authentication of Curcuma longa adulterated with C. heyneana. J Appl Res Med Aromat Plants 13: 100203. DOI: 10.1016/j.jarmap.2019.100203.

Yang Y, Yue Y, Runwei Y, Guolin Z. 2010. Cytotoxic, apoptotic and antioxidant activity of the essential oil of Amomum tsao-ko. Bioresour Technol 101: 4205-4211. DOI: 10.1016/j.biortech.2009.12.131.

Zagaja M, Andres-Mach M, Patrzylas P, Pyrka D, Szpringer M, FlorekŁuszczki M, et al. 2016. Influence of xanthotoxin (8methoxypsoralen) on the anticonvulsant activity of various novel antiepileptic drugs against maximal electroshock-induced seizures in mice. Fitoterapia 115: 86-91. DOI: 10.1016/j.fitote.2016.09.020.

Zagaja M, Pyrka D, Skalicka-Wozniak K, Glowniak K, Florek-Luszczki M, Glensk M, et al. 2015. Effect of xanthotoxin (8-methoxypsoralen) on the anticonvulsant activity of classical antiepileptic drugs against maximal electroshock-induced seizures in mice. Fitoterapia 105: 1-6. DOI: $10.1016 /$ j.fitote.2015.05.020

Zhai B, Zhang N, Han X, Li Q, Zhang M, Chen X, et al. 2019. Molecular targets of $\beta$-elemene, a herbal extract used in traditional Chinese medicine, and its potential role in cancer therapy: A review. Biomed Pharmacother 114: 108812. DOI: 10.1016/j.biopha.2019.108812.

Zhang X, Wei C, He L, An J. 2018. Xanthotoxin induces apoptosis in SGC-7901 cells through death receptor pathway. Chinese Herb Med 10: 437-444. DOI: 10.1016/j.chmed.2018.08.009. 